Review Article

\title{
Comparative Analysis of the Omics Technologies Used to Study Antimonial, Amphotericin B, and Pentamidine Resistance in Leishmania
}

\author{
Gagandeep Kaur and Bhawana Rajput \\ University Avenue, College of Medical, Veterinary and Life Sciences, University of Glasgow, Lanarkshire G12 8Q, UK \\ Correspondence should be addressed to Gagandeep Kaur; gagandeep_kaur87@live.com
}

Received 20 December 2013; Revised 24 April 2014; Accepted 28 April 2014; Published 12 May 2014

Academic Editor: C. Genchi

Copyright (C) 2014 G. Kaur and B. Rajput. This is an open access article distributed under the Creative Commons Attribution License, which permits unrestricted use, distribution, and reproduction in any medium, provided the original work is properly cited.

Leishmaniasis is a serious threat in developing countries due to its endemic nature and debilitating symptoms. Extensive research and investigations have been carried out to learn about the mechanism of drug resistance in Leishmania but results obtained in the laboratory are not in agreement with those obtained from the field. Also the lack of knowledge about the mode of action for a number of drugs makes the study of drug resistance more complex. A major concern in recent times has been regarding the role of parasitic virulence in drug resistance for Leishmania. Researchers have employed various techniques to unravel the facts about resistance and virulence in Leishmania. With advent of advanced and more specific means of detection, further hints about probable mechanisms of conferring resistance are expected. This review aims to provide a consolidated picture along with a comparative account of the work done so far to study the mechanism of antimony, amphotericin B, and pentamidine resistance using various techniques.

\section{Leishmaniasis: Incidence, Cause, and Resistance}

Leishmaniasis forms the ninth largest disease burden in the world affecting more than 90 countries on every continent except Antarctica and Australia (see http://www.cdc. gov/parasites/leishmaniasis/epi.html). It is an endemic disease with an estimated 12 million patients currently and a global rise of up to 2 million patients annually (see http://www.who.int/leishmaniasis/en/).

The principal cause of the disease is Leishmania parasite. On the basis of symptoms, leishmaniasis can be classified into two forms-cutaneous and visceral. L. donovani, L. infantum, and $L$. chagasi are the causative agents of visceral leishmaniasis (VL). This form of the disease is characterized by fever, weakness, night sweats, hepatomegaly, or splenomegaly and is mainly reported in regions of India, Bangladesh, Sudan, Ethiopia, and Brazil. Cutaneous leishmaniasis (CL), on the other hand, arises as a sore at the site of insect bite which can proceed to a severe form at times. CL can take two forms: diffuse CL or mucocutaneous leishmaniasis. In diffuse CL, skin lesions are widespread on the body that resemble leprosy. Mucocutaneous leishmaniasis begins with ulceration in the nares that proceeds further to nasal septum, pharynx, or larynx. It can eventually lead to remarkable disfigurement in the patient. It is normally reported in Africa, Latin America, and Middle East. The causative agents for CL have been classified as old and new world species as follows $[1,2]$ :

old world CL: L. major, L. tropica, L. (L.) aethiopica, and L. infantum;

new world CL: $L$. (L.) mexicana, $L$. (L.) amazonensis, L. braziliensis, $L$. (V.) panamensis, $L$. (V.) peruviana, $L$.

(V.) guyanensis, L. (L.) pifanoi, L. (L.) venezuelensis, $L$. (L.) shawi, and L. (V.) lainsoni.

Female sand fly acts as a carrier to transmit the diseasecausing parasite into the host. Basically, the parasite enters the body of the host in metacyclic promastigote form. Thereafter, it transforms and multiplies into amastigote form [2]. Over 
TABLE 1: Drugs used for the treatment of leishmaniasis.

\begin{tabular}{|c|c|c|c|c|c|}
\hline Serial number & $\begin{array}{l}\text { Name of the } \\
\text { drug }\end{array}$ & Mode of action & $\begin{array}{c}\text { Mode of } \\
\text { administration } \\
\end{array}$ & Adverse effects & References \\
\hline 1 & $\begin{array}{l}\text { Pentavalent } \\
\text { antimonials }\end{array}$ & $\begin{array}{l}\text { Inhibition of glycolysis and } \\
\beta \text {-oxidation of fatty acids of } \\
\text { parasite }\end{array}$ & $\begin{array}{l}\text { Intralesional for CL } \\
\quad \text { Parenteral }\end{array}$ & $\begin{array}{c}\text { Abdominal pain, erythema, nausea, } \\
\text { toxicity (hepatic, pancreas, renal, } \\
\text { muscular, and skeletal } \\
\text { cardiothrombocytopenia or } \\
\text { leukopenia) }\end{array}$ & {$[2,60,61]$} \\
\hline 2 & Amphotericin B & $\begin{array}{c}\text { Binding to parasite's } \\
\text { membrane sterols and } \\
\text { changing its permeability } \\
\text { selective to } \mathrm{K}^{+} \text {and } \mathrm{Mg}^{+}\end{array}$ & $\begin{array}{l}\text { Liposomal } \\
\text { formulations } \\
\text { Deoxycholate } \\
\text { formulations }\end{array}$ & $\begin{array}{l}\text { Fever, nausea, hypokalemia, } \\
\text { anorexia, leukopenia, kidney } \\
\text { failure, and heart problems }\end{array}$ & {$[2,59-61]$} \\
\hline 3 & Pentamidine & $\begin{array}{l}\text { Interferes with DNA } \\
\text { synthesis and modifies the } \\
\text { morphology of kinetoplast }\end{array}$ & $\begin{array}{c}\text { Parenteral } \\
\text { Intramuscular } \\
\text { administration }\end{array}$ & $\begin{array}{c}\text { Pain, nausea, vomiting, dizziness, } \\
\text { myalgia, hypertension, headache, } \\
\text { hypoglycemia, and transient } \\
\text { hyperglycemia }\end{array}$ & {$[1,2,60,61]$} \\
\hline 4 & Miltefosine & $\begin{array}{c}\text { Associated with } \\
\text { phospholipid biosynthesis } \\
\text { and alkyl-lipid metabolism } \\
\text { in Leishmania }\end{array}$ & Oral for VL & $\begin{array}{l}\text { Nausea, vomiting, diarrhea, and } \\
\text { raised creatinine }\end{array}$ & {$[1,2,59,60]$} \\
\hline 5 & Paromomycin & $\begin{array}{c}\text { Inhibition of protein } \\
\text { biosynthesis in sensitive } \\
\text { organism }\end{array}$ & $\begin{array}{l}\text { Topical for CL } \\
\text { Parenteral for VL }\end{array}$ & $\begin{array}{l}\text { Erythema, pain, oedema, and } \\
\text { ototoxicity (damage to internal ear) }\end{array}$ & {$[1,2,60]$} \\
\hline
\end{tabular}

the years, a number of drugs have been employed for the treatment of the disease amongst which antimony (Sb) containing compounds called antimonials are the most preferred drugs worldwide. A brief account about the mechanism of action and mode of administration of these drugs has been presented in Table 1.

The efficiency of these drugs depends upon
(a) immune status of the host,
(b) parasite factors,
(c) drug pharmacokinetics [1,3].

But despite so many treatment options, "drug resistance," especially antimonial resistance, is a serious problem associated with Leishmania research. The seriousness of the issue can be assessed from the fact that there are regions in the world that have been reported of being completely resistant towards therapeutics. One such region is that of Bihar (India) which has been reported to be unresponsive towards pentavalent antimonial treatment [3]. Moreover, resistant strains for almost all existing drugs can be obtained under laboratory conditions [4-8]. Interestingly, there are instances of varying virulence in Leishmania parasite on attainment of drug resistance [9-12]. This supports the view that there exists a relation between virulence and drug resistance in Leishmania. Some scientists are of an opinion that drug resistance comes with a fitness cost. However, not much work has been conducted to relate drug resistance with virulence in order to scientifically support this hypothesis [12].

The present paper aims to provide an overall picture of the techniques used so far to study the area of drug resistance in Leishmania taking three major drugs (antimonials, amphotericin $\mathrm{B}$, and pentamidine) into account and to highlight the advantages and drawbacks of each. Although miltefosine is being used extensively for leishmaniasis, we have no reports of clinical incidences of resistance in miltefosine $[13,14]$. Thus not much is spoken about miltefosine and its drug resistance in this review.

\section{Antimonial Resistance in Leishmania}

Pentavalent antimonials ( $\mathrm{Sb}(\mathrm{V})$ ) are the first line of treatment against leishmaniasis. Studies suggest that antimonials inhibit vital metabolic processes like fatty acid oxidation, glycolysis, and energy metabolism [13]. But the exact mode of action for the drug is still unknown. To add to the trouble is the problem of "drug resistance." Many attempts have been made to study the mechanism of drug resistance in Leishmania but the true phenomenon still remains obscure. The major breakthroughs in this field have been discussed in the following paragraphs.

\subsection{Advances to Study Mechanism of Antimony Resistance}

2.1.1. Gene Amplification (Figure 1). Genomic studies have revealed gene amplification to be one of the major mechanisms of attaining drug resistance in Leishmania. Genes that have been found to be amplified are MRPA and gsh1 $[15,16]$ and the genomic results have been confirmed through various proteome analyses studies (RT-PCR, and SILAC) as well [17-19]. MRPA gene codes for an ABC transporter that helps in sequestration of metal-thiol complex in an intracellular organelle which ultimately leads to removal of drug molecules from the cytoplasm [15]. Similarly gsh1 gene codes for the heavy subunit of $\Upsilon$-GCS which is a rate-limiting 
enzyme of glutathione (GSH) biosynthetic pathway that helps in drug detoxification [16].

However, when similar studies were repeated with clinical isolates of $L$. donovani, no noticeable change in expression levels of MRPA and gsh1 genes was established [20]. It was hypothesized that mutation in Leishmania parasite is speciesspecific which results in such contradictions. In recent times, with the advent of next-generation sequencing, this hypothesis could be justified to a certain extent. In fact results obtained from a recent whole genome comparison study conducted on S- and R-strains of Leishmania support the fact that the genetic background of the parasitic strain could be the chief reason for the prevalent heterogeneity amongst antimony-resistance phenotypes [21].

\subsubsection{Cellular Sequestration or Efflux of Metal-Thiol Conjugate} from the Cell (Figure 1). Genomic comparisons of resistant and sensitive strains of Leishmania point towards the overexpression of genes like ODC (ornithine decarboxylase), SAHH, and FPGS in resistant lines [15, 22]. ODC is essential for trypanothione synthesis which is a major thiol for formation of metal-thiol complex thus leading to cellular extrusion of drug molecules [22]. Enhanced levels of thiol metabolising enzymes and oligopeptidase $\mathrm{B}(O P B)$ have been observed in resistant lines after 2-DE and LC-ES MS/MS comparisons. $O P B$ has been found to play a role in immune evasion, fitness, and virulence in L. donovani and L. major [23]. In fact metabolomic studies performed on resistant strains have also observed manyfold increases in the thiol level of resistant parasite [24].

Studies have proven the importance of ODC overexpression and thiol-conjugated efflux pumps in antimony resistance in various leishmanial strains [17, 25]. A recent study has found a new half-transporter, $\mathrm{ABC14}$, to be involved in antimony resistance. $\mathrm{ABC} 14$ has been found to be responsible for the efflux of antimony from antimony-resistant cells of L. major in the form of a drug-thiol conjugate [25]. These findings support the role of cellular sequestration and subsequent efflux of drug from the parasitic cytoplasm in the form of metal-thiol conjugate.

2.1.3. Decreased Activation of Antimony Prodrug (Figure 1). It is not known whether activation of antimony prodrug, that is, conversion of $\mathrm{Sb}(\mathrm{V})$ to $\mathrm{Sb}$ (III), occurs intracellularly or extracellularly. Yet it is evident that hindrance in activation of $\mathrm{Sb}$ prodrug forms the first means of drug resistance in Leishmania. An intracellular enzyme, TDR1, has been identified that helps in the conversion of $\mathrm{Sb}(\mathrm{V})$ to $\mathrm{Sb}$ (III) and is believed to be underexpressed in R-cells [26].

\subsubsection{Decreased Uptake of Drug by Underexpression of AQP1} (Figure 1). Gourbal et al. showed that overexpression of AQP1 (aquaglyceroporin) gene in resistant strains of $L$. tarentolae, $L$. infantum, and $L$. major results in increased sensitivity of the cells towards trivalent antimonial (Sb (III)) drug [27]. Various studies conducted in recent times confirm these findings $[17,18]$. It is thus known that downregulation of $A Q P 1$ plays a significant role in antimony resistance for Leishmania as it prevents the entry of Sb (III) into the parasitic cell.

Recent Findings. Recently certain new mechanisms like decreased DNA fragmentation and programmed cell death have been identified to confer Sb resistance in Leishmania [20].

Additionally sequencing studies are revealing newer mechanisms of resistance. For instance, a novel terminal deletion of chromosome 31 has been reported recently amongst three antimony-resistant mutants of $L$. major through nextgeneration sequencing and comparative genomic hybridization (CGH) [28].

Comparative proteome analysis of R- and S-strains of Leishmania has been conducted in various studies. These studies revealed overexpression of metabolically significant proteins like carboxypeptidase, enolase, fructose-1, 6bisphosphatase, $H s p 70$, and $H s p 83$ and downregulation of a kinetoplast membrane protein (KMP11) and calcineurin in Rstrains [29-31].

Hsp70 has already been known to have an immunestimulatory role in L. infantum and demands further research to indicate its importance in drug resistance. Proteome comparison, DNA fragmentation assays, and mitochondrial membrane potential analysis of two strains of $L$. donovani demonstrated overexpression of Hsp83 and reduction in DNA fragmentation and membrane potential of resistant parasites [20,29].

KMP11 belongs to a highly conserved family of membrane glycoproteins which are said to define virulence in Leishmania [30].

Downregulation of calcineurin has been reported for the first time in literature. It is a calcium dependent protein phosphatase involved in varied cellular activities like cell survival and apoptosis. It is thus believed that underexpression of calcineurin can protect resistant parasites from antimonyinduced apoptosis [31], although further work needs to be done in this area.

Full metabolome comparisons of sensitive and resistant clinical isolates of $L$. donovani have identified a number of variations between the two phenotypes [32-34]. Major difference has been found in the lipid composition of the two phenotypes. Decreased level of sphingolipids and sphingomyelins and increased levels of phosphatidylcholine and unsaturated fatty acids were observed for resistant strain which indicates involvement of changed membrane fluidity and characteristics for conferring $\mathrm{Sb}$ resistance [32].

Levels of surface glycoconjugates were found to be enhanced in R-strains. These glycoconjugates have been known to play some role in metacyclogenesis and virulence of the parasite [33].

A recent metabolomics comparison between resistant and sensitive strains of $L$. infantum promastigotes using CE-ESI-TOF-MS exhibited variations in levels of various amino acids, which were not shown in previous studies [35]. Another study showed the increase in metabolites involved in urea cycle and cysteine transsulfuration pathways [34]. Such results are thus encouraging for the future of drug resistance 


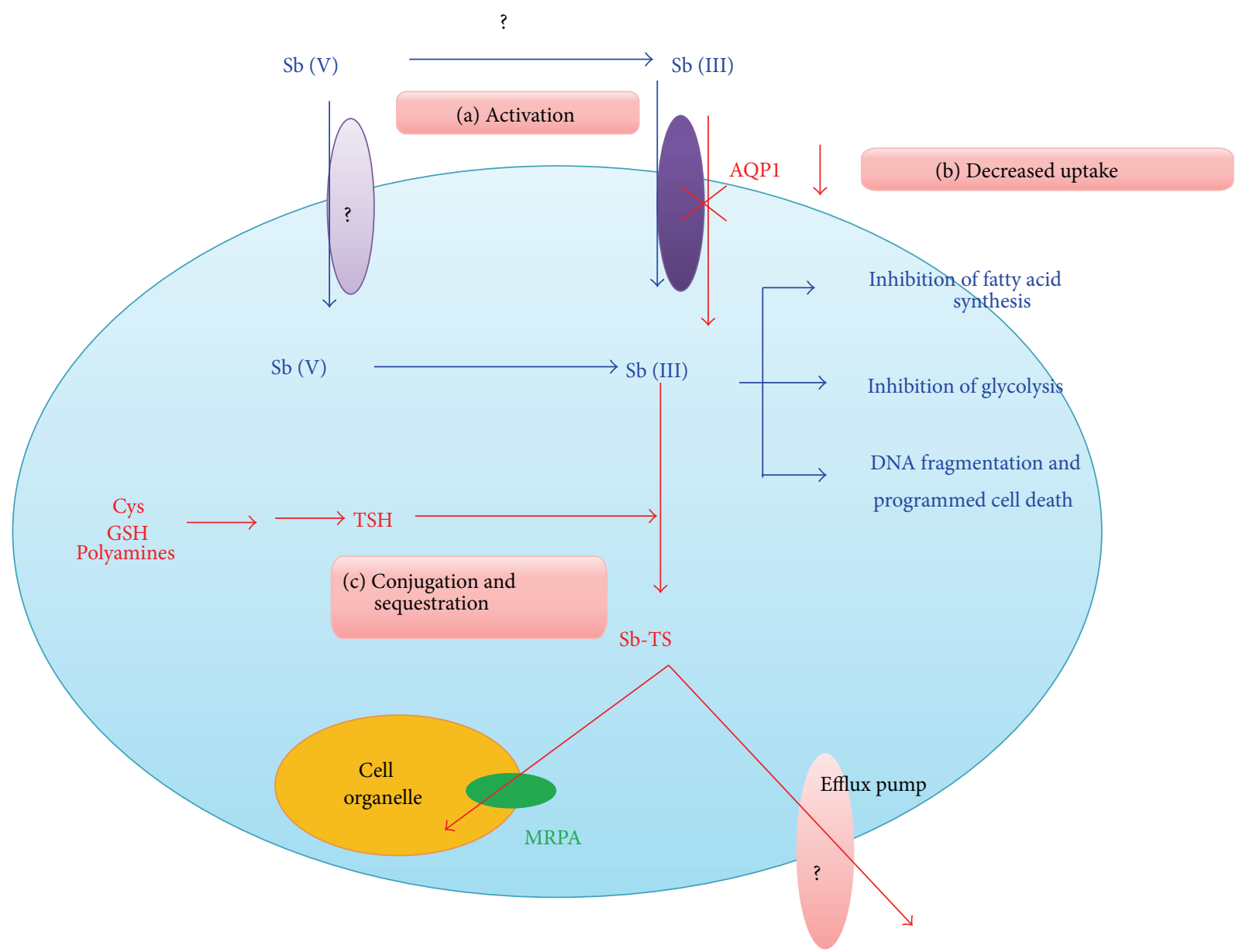

FIgure 1: The mechanisms of antimony resistance in Leishmania. (a) Activation: conversion of Sb (V) to Sb (III) is inhibited in R-cells. This inhibition can either occur extracellularly (by inactivation of enzymes still unknown) or intracellularly (by inhibition of enzymes like ACR2 or TDR1). (b) Decreased uptake: decreased expression of AQP1 reduces Sb uptake into the cell thus conferring resistance. (c) Conjugation and sequestration: increased thiol levels (like cysteine (Cys), GSH, TSH, and polyamines) in the cell result in its conjugation with antimony to form antimony-thiol complex (Sb-TS) which then results in sequestration of the complex into a cell organelle or extrusion from the cell thus lowering intracellular amount of antimony. Blue lines indicate the probable drug action in sensitive Leishmania strains while red lines depict the probable routes to achieve resistance as observed in resistant cells.

studies and demand further exploration in this area with the help of modern analytical techniques.

\section{Amphotericin B Resistance in Leishmania}

Amphotericin $\mathrm{B}(\mathrm{AmB})$ is the second line of defence against leishmaniasis [36]. This polyene drug has high affinity towards membrane-bound ergosterol which leads to the formation of small membranous pores that alters membrane permeability towards cations, water, and glucose molecules [5]. Consequently, osmotic integrity of the cell is disrupted causing leakage of magnesium and potassium ions that finally leads to cell death [37].

$\mathrm{AmB}$ resistance in field or clinical isolates is not common for Leishmania. Several studies prove instead that AmB susceptibility remains unaffected even after repeated administration of the drug $[38,39]$. Although for patients with HIV/VL coinfections the relapse rate after AmB treatment is quite high which might eventually lead to resistance [39]. A recent report from India has identified a patient infected by $\mathrm{AmB}$ resistant strain of $L$. donovani which presents the possibility of emergence of other such cases in future [40].

\subsection{Advances to Study Mechanism of AmB Resistance}

3.1.1. Change in Membrane Fluidity (Figure 2). AmB toxicity relies on the interaction of the drug with parasitic membrane. Hence preliminary studies about AmB resistance relied on flow cytometric assays to investigate membrane potential of Leishmania cells [39]. Mbongo et al. (1997) used MS analysis to prove that AmB-resistant promastigotes of $L$. donovani are rich in cholesta-5,7,24-trien-3 $\beta$-ol (a precursor in ergosterol biosynthesis) which results in increased membrane fluidity. Furthermore the intracellular AmB concentration was found to be low for resistant cells [5]. GC-MS studies for sterol analysis proved abundance of methylcholesta sterol 


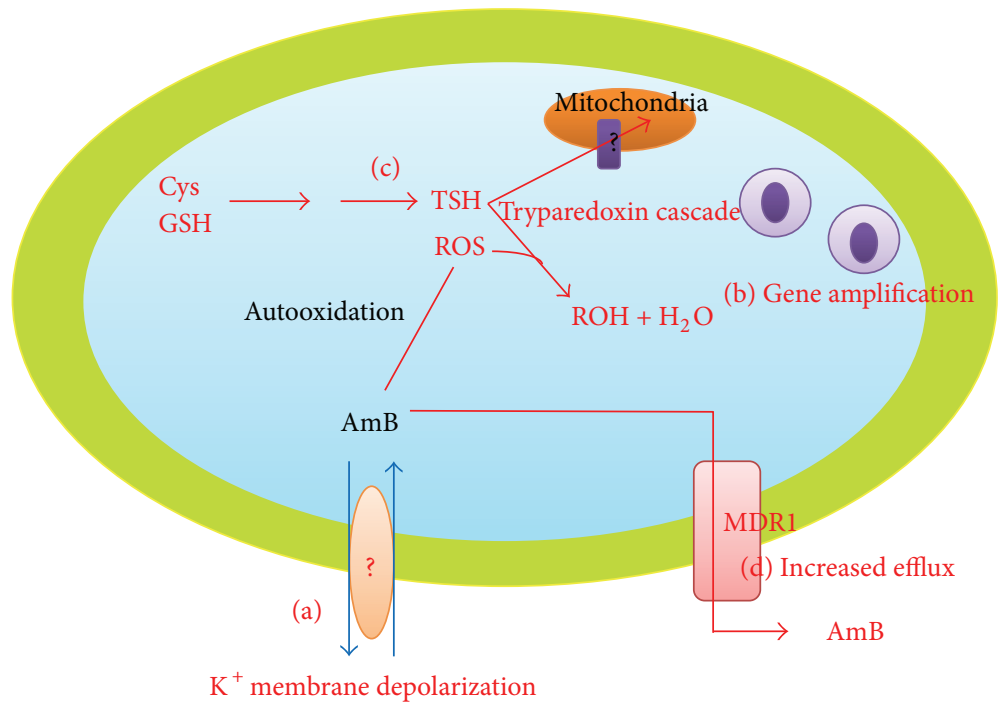

FIGURE 2: The mechanisms of amphotericin B resistance in Leishmania. (a) Change in membrane fluidity results in blocking of the drug entry inside the cell. The membrane transporters or factors responsible for such changes in membrane depolarization are still unknown. (b) Gene amplification of genes to confer resistance. (c) Activation of tryparedoxin cascade to prevent the oxidative damage caused by the drug. (d) Drug efflux through various membrane-bound pumps like MDR1.

in resistant promastigotes and amastigotes under in vitro conditions. Interestingly in vivo studies performed with the wild-type and drug-resistant amastigotes and promastigotes revealed decreased infectivity in drug-resistant promastigotes and inability to cause infection in resistant amastigotes [4]. The exact reason for this decreased infectivity is not known, but it is reasoned out that decreased microviscosity of membrane in resistant parasites might render membrane receptors nonfunctional which affects the infectivity [5].

\subsubsection{Decrease in Thiol and ROS Levels and Increase in Drug} Efflux from the Cell (Figure 2). Recently the mechanism of $\mathrm{AmB}$ resistance in clinical isolates of $L$. donovani was studied where an increase in membrane fluidity and decrease in potassium leakage were observed for R-strains. The intracellular thiol and ROS (reactive oxygen species) level was low in resistant cells which proved that tryparedoxin cascade might be operative to prevent oxidative damage by elimination of toxic peroxides from the cells [41].

Furthermore, RT-PCR results of the clinical isolates of AmB-resistant strains showed the upregulation of genes involved in trypanothione biosynthesis and tryparedoxin cascade. The mRNA level of MDR1 (ABC transporter) was found to be 4 -fold higher in R-strains which supported the observations for increased drug efflux. Finally the expression of SCMT, an enzyme responsible for C24 transmethylation in sterol biosynthetic pathway, was tested. It has two transcripts-SCMT A and SCMT B. Expression profiling showed lack of expression for SCMT A and increased expression for SCMT B. Altered expression of SCMT genes in case of resistance supported the view that defective expression of enzymes involved in sterol biosynthetic pathway leads to absence of membrane ergosterol in R-cells [41]. Further studies on SCMT and other enzymes involved in sterol biosynthesis could be expected to provide some results regarding drug resistance.

3.1.3. Gene Amplification in Extrachromosomal Circle (Figure 2). An alternative mechanism for drug resistance was tested in L. tarentolae. Gene amplification studies for resistance identified two strains exhibiting amplification in extrachromosomal DNA of the parasite. The in vitro resistance levels were found to be directly proportional to the copy number of amplicons and the resistance achieved was highly stable [42]. However, the exact region undergoing amplification still remains unknown.

SDS-PAGE results of a recent case report from India revealed overexpression of proteins in the range of 65 to $80 \mathrm{kDa}$, in AmB-resistant sample. This band was recognised to be cysteine protease. But no known mechanistic significance of this protein has been reported for drug resistance in Leishmania to date [40]. Again this is just a case report and this data cannot be completely relied upon as it could be just an individualistic observation.

\section{Pentamidine Resistance in Leishmania}

Pentamidine (PMD) has been used as an alternative treatment for $\mathrm{VL}$ in cases of $\mathrm{Sb}$ resistance. Its exact mode of action is unknown but reports show that it inhibits activity of enzyme S-adenosyl-L-methionine decarboxylase, interferes with polyamine synthesis, and decreases mitochondrial membrane potential [43]. Thus the main target for the drug seems to be parasitic mitochondria [44]. Evidence of resistance against PMD has been reported but the mechanism of resistance is not understood properly $[45,46]$. 


\subsection{Advances to Study Mechanism of Pentamidine Resistance}

4.1.1. Change in kDNA Genome Sequence (Figure 3). Early experiments using molecular modelling, biophysical analysis, and molecular biology revealed the interaction of PMD with minor groove of AT-rich regions of DNA, that is, kDNA. Hence initial attempts about PMD resistance in Leishmania focused on kDNA comparisons. Southern blot comparisons between the kDNA of wild-type and PMD-resistant cells of L. donovani and L. amazonensis proved significant variations. Sequence homology result was very low $(32-51 \%)$ thus supporting the view about changes in $\mathrm{kDNA}$ sequence to confer resistance in these species [8].

4.1.2. Drug Efflux Pumps (Figure 3). In a study performed with L. major, role of a new gene named PRP1, coding for an $A B C$ transporter, was identified in drug resistance [47]. In vitro study performed on L. infantum exhibited that PRP1-transfected parasites possessed 3-fold resistance against PMD. Similar results were obtained for L. mexicana and L. amazonensis [44]. Hence it was confirmed that PRP1 plays a definite role for PMD resistance in Leishmania. Presence of other transporters having similar function is being hypothesized but none have yet been recognized [7].

4.1.3. Decreased Mitochondrial Uptake (Figure 3). Pentamidine accumulation in mitochondria is a reason for its toxicity to parasitic cells. Drug uptake studies showed that the mitochondrial uptake of pentamidine is inhibited in resistant cells which lead to rapid removal of the drug present in the cytosol [48]. Another study performed on L. amazonensis indicated modified uptake of spermidine and putrescine in PMDresistant parasites. Pentamidine uptake by resistant clones was shown to be carrier mediated and energy dependent $[43,49]$. The involvement of polyamine biosynthetic pathway in pentamidine resistance was further studied and it was found that intracellular ornithine and arginine levels increase while those of putrescine decrease for PMD-resistant $L$. donovani and L. amazonensis cells [49]. It has been known that pentamidine competitively inhibits arginine transport and noncompetitively inhibits spermidine and putrescine transport. Hence increased arginine level and decreased putrescine level are a mechanism to prevent the uptake of pentamidine by the mitochondria. Resistant cells show lowered mitochondrial membrane potential which again help in drug exclusion. Biochemical studies have indicated reduced mitochondrial uptake of PMD in resistant cells. It has been shown that administration of mitochondrial metabolic inhibitors like sodium azide and KCN lowered the PMD uptake thus highlighting its significance as resistance mechanism. However results with P-glycoprotein pump inhibitors (verapamil) varied. While, on one hand, verapamil reversed PMD resistance phenotype for L. mexicana, on the other hand no such effect was shown for L. donovani [50]. This proves that drug resistance in Leishmania is affected by species factors as well.

Various enzyme assays have proved that the levels of enzyme involved in polyamine biosynthesis are altered in
PMD-resistant parasites. The level of ODC (ornithine decarboxylase), which converts ornithine to putrescine, is lowered in PMD-resistant cells which accounts for the decreased levels of putrescine in resistant cells [51]. The activity of most enzymes involved in maintenance of mitochondrial potential has also been found to be diminished in R-strains thus leading to lowered membrane potential [50].

\section{Virulence and Drug Resistance: Are They Related?}

"Virulence," "fitness," and "parasitic proficiency" are different terms used to describe the set of characteristics present in a parasite to divide and transmit disease. Studies on viruses have pointed towards a decrease in viral replication and differentiation on attaining resistance towards antiretroviral drugs. A similar observation is expected for Leishmania as well [12]. In fact, numerous in vitro studies prove this thought to be true.

In a report about antimony resistance in American species of Leishmania (L. V. guyanensis, L. L. amazonensis, and L. V. Braziliensis) it was seen that resistant parasites had reduced infectivity as compared to their progenitor [52]. Similar results are available in ricin-resistant L. major, glucantime-resistant L. (V) guyanensis, and AmB-resistant $L$. mexicana [12]. However after subsequent passages in drug absence, the resistant strains were found to be able to grow faster which proved the existence of some compensatory mutation in resistant strains. Thus this led towards deduction of a hypothesis that drug-resistant parasite is likely to be less virulent than wild type. Consequently it was believed that fitness cost can be used as a means to fight leishmaniasis [52]. However, in vivo models displayed a different picture altogether. While hypothesizing about decreased fitness of resistant leishmaniasis cells, an important fact about the simplicity of in vitro models was neglected. In reality drug resistance is not so simple and is rather achieved via a complex interplay of immune response, nutrient availability, and oxidative stress imposed by environment on Leishmania.

Leishmania is a highly adaptive parasite. Over thousands of years, it has developed mechanisms to overcome the hostile environment encountered in insect's midgut and host's macrophages [11]. Thus it is not hard to believe that it can develop drug resistance without paying a fitness cost in return. In a study relating metacyclogenesis (parameter for infectivity) to antimony resistance in L. donovani it was established that capacity of differentiation was significantly higher in antimony-resistant cells [10]. Another report about clinical isolates taken from Nepalese strains of L. donovani showed that resistant cells had the ability to attain a higher parasite density, contained a higher number of metacyclics, and possessed increased capacity of in vivo infection than sensitive cells. [11]. Further work done by the same group of researchers successfully proved that resistant strains of L. donovani have higher virulence and disease burden as compared to sensitive ones with the help of in vivo models [53]. These reports prove that resistance mechanisms derived in the field are quite different from the one observed under 


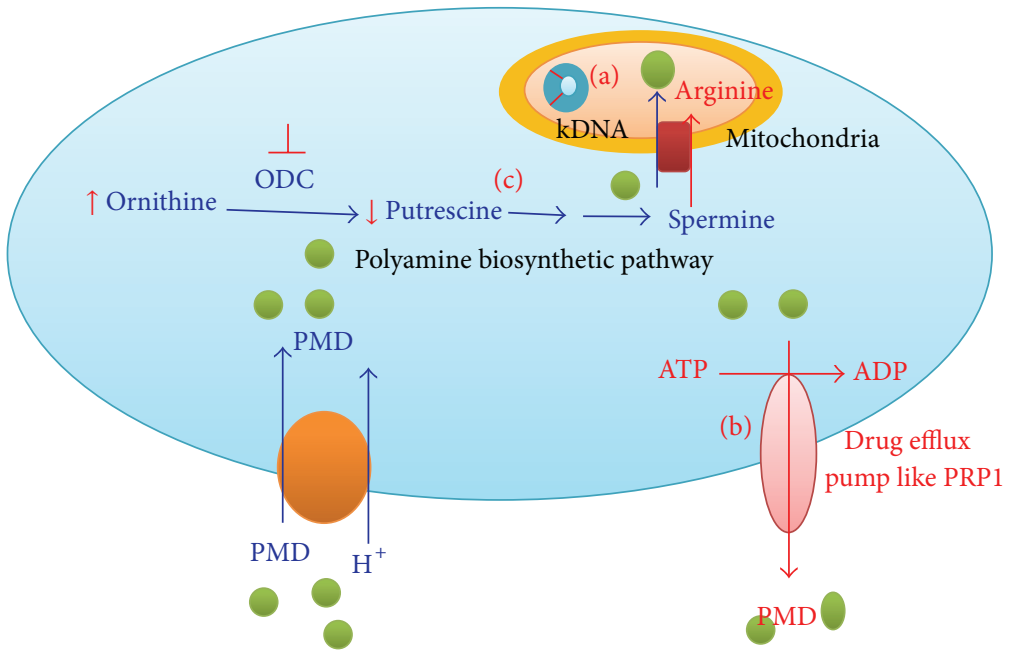

FIGURE 3: The mechanisms of pentamidine resistance in Leishmania. (a) Change in kDNA sequence confers resistance. The exact mechanism of conferring resistance remains unknown. (b) Presence of drug efflux pumps like PRP1 to remove the drug molecules from the cell and thus protect it from damage. (c) Reduced uptake of pentamidine in mitochondria due to altered polyamine biosynthetic pathways and lowered membrane potential. Blue lines indicate the probable drug action in sensitive Leishmania strains while red lines depict the probable routes to achieve resistance as observed in resistant cells.

laboratory conditions [54]. Moreover it even questions the authenticity of the research done so far regarding drug resistance, as most of the studies are conducted under in vitro conditions.

The other question which lingers in mind is about the cause of the increased infectivity in field isolates. A hint about the probable reason was provided during metabolomics comparison between clinical isolates of resistant and sensitive $L$. donovani parasites. This group observed an increase in the amount of surface glycoconjugates in resistant strain. It is known that in Leishmania glycosylated proteins and proteoglycans are associated with metacyclogenesis and thus in turn with virulence [33]. So the question arises, is this increase in surface glycosylation a means to improve cell proficiency? And this is not a solo report as it has been stated that changes in metabolic pathways involving pteridine reductase and trypanothione reductase play a role in attaining resistance in Leishmania. These have an intimate relationship with parasite's virulence and can be considered to play a specific role in affecting infectivity. Hence it could be considered that such metabolic parameters might be able to predict changes in cell proficiency associated with drug resistance. However, the true nature of this association is still unknown [12].

These findings about increased infectivity of drugresistant Leishmania parasites are an alarm signal. To date we have studied the two phenomena-virulence and drug resistance-separately for Leishmania. But recent reports prove the close association between the two. It is thus necessary to design future studies to predict effects of drug resistance in virulence for Leishmania under in vivo conditions.

\section{Concluding Remarks and Future Prospects}

Drug resistance is a highly complex mechanism and holds a strong association with parasitic infectivity. Contrary to in vitro results, field isolates show increased virulence and carry a potential risk of selection of virulent pathogens through chemotherapeutic interventions. The three branches of biotechnology that have been used to date for the study of drug resistance are genomics, proteomics, and metabolomics. A comparative account of these techniques is given in Table 2.

Comparison of complete proteome and metabolome has been employed quite recently. But genomic analysis has been prevalent for a long time. Genomic data is able to provide evidence for the root cause of drug resistance phenotype in Leishmania. Also phylogenetic classification of parasitic species helps in prediction of gene function in related species. As such, out of 20 causative species of leishmaniasis only 7 are fully sequenced [55]. Comparison of the genomes of these species reveals high percentage identity. For instance, the average nucleotide identity between $L$. major and $L$. infantum is $94 \%$ (L. major and L. braziliensis is $77 \%$ and L. infantum and L. braziliensis is 77\%) [56]. But despite such high level of identity, the pathogenesis of each varies drastically. This either means that pathogenesis in Leishmania is determined by just a few species-specific genes or else genome plays a minor role in determining clinical outcome of the disease. Furthermore the sequence information obtained using Sanger sequencing does not provide the knowledge about gene copy number. Use of novel sequencing methods can overcome this shortcoming but at present this lack of data does not work in favour of the researchers [57].

Proteomics, on the other hand, focuses on the functional output of the cell. Essentially, it has been observed that 
TABLE 2: The advantages and disadvantages of strategies used to study drug resistance.

\begin{tabular}{|c|c|c|c|c|}
\hline & Techniques & Advantages & Disadvantages & References \\
\hline Genomics & $\begin{array}{c}\text { Southern blot, } \\
\text { microarray, northern } \\
\text { blot, sequencing, } \\
\text { PFGE, FIGE }\end{array}$ & $\begin{array}{l}\text { (a) Genetic basis of observed } \\
\text { phenotype } \\
\text { (b) Large genome coverage } \\
\text { (22-97.5\%) } \\
\text { (c) Easy to perform and interpret } \\
\text { (d) Reproducible }\end{array}$ & $\begin{array}{l}\text { (a) Not much differences amongst } \\
\text { species at genome level } \\
\text { (b) Not much informative } \\
\text { (c) Gene functions for most genes } \\
\text { still unknown. }\end{array}$ & $\begin{array}{c}{[7,8,14,15,22,37,} \\
41,42,44,47,51,62]\end{array}$ \\
\hline Proteomics & $\begin{array}{l}\text { 2DE, MALDI-TOF, } \\
\text { LC-MS/MS, } \\
\text { LC-ESI-MS/MS, } \\
\text { western blot, } \\
\text { immunoblot }\end{array}$ & $\begin{array}{l}\text { (a) Functional output of the cell } \\
\text { (b) Posttranslational changes } \\
\text { visualised } \\
\text { (c) Mostly automated } \\
\text { (d) Good indicator of protein } \\
\text { abundance and expression }\end{array}$ & $\begin{array}{l}\text { (a) Number of proteins lack } \\
\text { annotated functions } \\
\text { (b) Less abundant proteins hard to } \\
\text { detect } \\
\text { (c) Results from in vivo amastigotes } \\
\text { difficult to interpret } \\
\text { (d) Not reproducible in some cases }\end{array}$ & $\begin{array}{c}{[6,20,23,29,30} \\
40,49,51,51]\end{array}$ \\
\hline Metabolomics & $\begin{array}{l}\text { CE-ESI-TOF-MS, } \\
\text { HPLC, MALDI-TOF, } \\
\text { flow cytometry, } \\
\text { GC-MS }\end{array}$ & $\begin{array}{l}\text { (a) Closest correlation to } \\
\text { phenotype } \\
\text { (b) Rapid visualisation and } \\
\text { prediction of biological impact } \\
\text { (c) High mass accuracy } \\
\text { (d) Highly specific }\end{array}$ & $\begin{array}{l}\text { (a) Unable to quantify most of the } \\
\text { metabolites. } \\
\text { (b) Analyte derivatives make data } \\
\text { complex } \\
\text { (c) Data hard to analyse } \\
\text { (d) Complicated bioinformatics } \\
\text { tools needed } \\
\text { (e) Costly instrumentation }\end{array}$ & $\begin{array}{c}{[4,5,22,24,32,35} \\
\quad 41,43,48,51]\end{array}$ \\
\hline
\end{tabular}

PFGE: pulsed field gel electrophoresis; FIGE: field inversion gel electrophoresis; 2DE: two-dimensional gel electrophoresis; MALDI-TOF MS: matrix assisted laser desorption/ionisation time of flight mass spectrometry; LC-MS/MS: liquid chromatography mass spectra/mass spectrometry; LC-ESI-MS/MS: liquid chromatography electrospray ionisation tandem mass spectrometry; CE-ESI-TOF-MS: capillary electrophoresis mass spectrometry coupled with electrospray ionisation mass spectrometry; HPLC: high performance liquid chromatography; GC-MS: gas chromatography mass spectrometry.

most of the regulation in Leishmania is obtained posttranslationally. Hence proteomic studies provide more knowledge about differences between resistant and sensitive parasites as compared to genomic data [58]. It is a good indicator about protein abundance and expression and can yield an insight into species differences, stage differentiation, drug resistance, and virulence [59]. However, on the downside, a number of differentially expressed proteins found after proteome comparison lack an annotated function [57]. Most of the gel-based proteome comparisons only highlight abundant proteins, whereas the not-so-abundant ones seem to be masked [58]. Hence further research needs to be done in characterizing unidentified proteins, not-so-abundant proteins, and proteins with unknown functions.

With the advent of highly specific detection methods, like LC-MS, Orbitrap, GC-MS, and so forth, metabolomics data appears to be highly beneficial for Leishmania research. Metabolic profiles can give closest correlation to the phenotype in organisms. Metabolic maps provide a rapid and efficient method to visualize the metabolic changes and consequently predict its biological impact. Also due to high mass accuracy of metabolite masses obtained using recent techniques, $a b$ initio extension of metabolic networks is possible. This can be used to make hypothetical connections and predict metabolic transformations related to observed mass peaks. But despite these advantages, still a lot of advancement is required in this field. The current use of LC-MS does not allow efficient quantification of all detected metabolites. Many of the signals thus obtained correspond to analyte derivatives like isotopes, fragments, adducts, and so forth. Biologically this holds no significance and needs to be filtered. Mass spectrometry results generate a large volume of data which cannot be handled and interpreted using the available bioinformatics tools. Thus newer and complicated database and bioinformatics tools need to be developed for handling, filtering, and processing the data with ease [57]. From a personal observation, it can be added though that studies based on two or more strategies are more informative than the ones based on a single strategy.

In the end it could be concluded that drug resistance in leishmaniasis is a major concern for doctors and researchers worldwide. Much work has been done to understand the mechanism of resistance for major drugs against Leishmania but not much attempt has been made to study virulence in drug resistance. In recent times this has grabbed the attention of the scientists and further work in this area might reveal significant facts. Genomic, proteomic, and metabolomic approaches used so far have been quite informative. Additionally with advancements in technology better and more effective means for detection and characterization of genes, proteins, and metabolites are available. It could thus be believed that, with combined effort in this area, we might manage to formulate newer drugs, identify good biomarkers, and develop effective vaccines to fight against this disease and eventually overcome the problem of drug resistance which is prevalent at present. 


\section{Glossary}

$A Q P 1$ gene: $\quad$ Aquaglyceroporin gene

Drug resistance: Reduction in effectiveness of a drug in curing a disease or condition

GC-MS: $\quad$ Gas chromatography-mass spectrometry

GSH: Glutathione

GSH1 gene: Gamma-glutamylcysteine synthetase gene

$\mathrm{kDa}$ :

kDNA:

MDR1: Kilo Dalton

MDR1:

Metacyclogenesis:

Kinetoplastid deoxyribonucleic acid Multidrug-resistant protein 1

Microviscosity: Friction experienced by a single

The differentiation of metacyclic promastigotes particle undergoing diffusion because of its interaction with its environment at the micrometre length scale

Pharmacokinetics: Branch of pharmacology concerned with the movement of drugs within the body

Prodrug: A biologically inactive compound that can be metabolized in the body to produce a drug

PRP1: Pentamidine resistance protein 1

Relapse rate: The rate of reemergence of disease symptoms in a patient after treatment

SCMT: S-Adenosyl-1-methionine: C-24-delta-sterol-methyltransferase

Sequence homology: Sequence similarity as a result of common ancestry

TDR1 enzyme: $\quad$ Thiol dependent reductase.

\section{Conflict of Interests}

The authors declare that there is no conflict of interests regarding the publication of this paper.

\section{Acknowledgments}

The authors would like to thank Dr. Richard Burchmore for his guidance and the lab members at the Functional Genomics Lab at the University of Glasgow for all the assistance. The authors would also like to thank Dr. J K Srivastava for reviewing the paper for them.

\section{References}

[1] H. Goto and J. A. Lindoso, "Current diagnosis and treatment of cutaneous and mucocutaneous Leishmaniasis," Expert Review of Anti-Infective Therapy, vol. 8, no. 4, pp. 419-433, 2010.

[2] H. W. Murray, J. D. Berman, C. R. Davies, and N. G. Saravia, "Advances in leishmaniasis," The Lancet, vol. 366, no. 9496, pp. 1561-1577, 2005.

[3] S. L. Croft, S. Sundar, and A. H. Fairlamb, "Drug resistance in Leishmaniasis," Clinical Microbiology Reviews, vol. 19, no. 1, pp. 111-126, 2006.
[4] H. I. Al-Mohammed, M. L. Chance, and P. A. Bates, "Production and characterization of stable amphotericin-resistant amastigotes and promastigotes of Leishmaniamexicana," Antimicrobial Agents and Chemotherapy, vol. 49, no. 8, pp. 3274-3280, 2005.

[5] N. Mbongo, P. M. Loiseau, M. A. Billion, and M. RobertGero, "Mechanism of amphotericin B resistance in Leishmaniadonovani promastigotes," Antimicrobial Agents and Chemotherapy, vol. 42, no. 2, pp. 352-357, 1998.

[6] J. Walker, J. J. Vasquez, M. A. Gomez et al., "Identification of developmentally-regulated proteins in Leishmaniapanamensis by proteome profiling of promastigotes and axenic amastigotes," Molecular and Biochemical Parasitology, vol. 147, no. 1, pp. 6473, 2006.

[7] A. C. Coelho, L. G. Gentil, J. F. da Silveira, and P. C. Cotrim, "Characterization of Leishmania (Leishmania) amazonensis promastigotes resistant to pentamidine," Experimental Parasitology, vol. 120, no. 1, pp. 98-102, 2008.

[8] M. Basselin, M.-A. Badet-Denisot, and M. Robert-Gero, "Modification of kinetoplast DNA minicircle composition in pentamidine-resistant Leishmania," Acta Tropica, vol. 70, no. 1, pp. 43-61, 1998.

[9] K. Ait-Oudhia, E. Gazanion, B. Vergnes, B. Oury, and D. Sereno, "Leishmania antimony resistance: what we know what we can learn from the field," Parasitology Research, vol. 109, no. 5, pp. 1225-1232, 2011.

[10] M. Ouakad, M. Vanaerschot, S. Rijal et al., "Increased metacyclogenesis of antimony-resistant Leishmaniadonovani clinical lines," Parasitology, vol. 138, no. 11, pp. 1392-1399, 2011.

[11] M. Vanaerschot, I. Maes, M. Ouakad et al., "Linking in vitro and in vivo survival of clinical Leishmaniadonovani strains," PLoS ONE, vol. 5, no. 8, Article ID e12211, 2010.

[12] S. Natera, C. Machuca, M. Padrón-Nieves, A. Romero, E. Díaz, and A. Ponte-Sucre, "Leishmania spp.: proficiency of drug-resistant parasites," International Journal of Antimicrobial Agents, vol. 29, no. 6, pp. 637-642, 2007.

[13] J. Chakravarty and S. Sundar, "Drug resistance in Leishmaniasis," Clinical Microbiology Reviews, vol. 19, no. 1, pp. 111-126, 2010.

[14] M. Berg, A. Mannaert, M. Vanaerschot et al., "(Post-) Genomic approaches to tackle drug resistance in Leishmania," Parasitology, vol. 140, no. 12, pp. 1492-1505, 2013.

[15] K. El Fadili, N. Messier, P. Leprohon et al., "Role of the ABC transporter MRPA (PGPA) in antimony resistance in Leishmaniainfantum axenic and intracellular amastigotes," Antimicrobial Agents and Chemotherapy, vol. 49, no. 5, pp. 1988-1993, 2005.

[16] K. Grondin, A. Haimeur, R. Mukhopadhyay et al., "Co- amplification of the $\gamma$-glutamyl cysteine synthetase gene gshl and of the $\mathrm{ABC}$ transporter gene pgpA in arsenite-resistant Leishmania tarentolae," The EMBO Journal, vol. 16, no. 11, pp. 3057-3065, 1997.

[17] S. Rai, Bhaskar, S. K. Goel et al., "Role of efflux pumps and intracellular thiols in natural antimony resistant isolates of Leishmaniadonovani," PLoS ONE, vol. 8, no. 9, Article ID e74862, 2013.

[18] M. Brotherton, S. Bourassa, P. Leprohon et al., "Proteomic and genomic analyses of antimony resistant Leishmaniainfantum mutant," PLoS ONE, vol. 8, no. 11, Article ID e81899, 2013.

[19] R. L. do Monte-Neto, A. C. Coelho, F. Raymond et al., "Gene expression profiling and molecular characterization of antimony resistance in Leishmaniaamazonensis," PLoS Neglected Tropical Diseases, vol. 5, no. 5, Article ID e1167, 2011. 
[20] B. Vergnes, B. Gourbal, I. Girard, S. Sundar, J. Drummelsmith, and M. Ouellette, "A proteomics screen implicates HSP83 and a small kinetoplastid calpain-related protein in drug resistance in Leishmaniadonovani clinical field isolates by modulating drug-induced programmed cell death," Molecular and Cellular Proteomics, vol. 6, no. 1, pp. 88-101, 2007.

[21] S. Decuypere, M. Vanaerschot, K. Brunker et al., "Molecular mechanisms of drug resistance in natural Leishmania populations vary with genetic background," PLoS Neglected Tropical Diseases, vol. 6, no. 2, Article ID e1514, 2012.

[22] A. Mukherjee, P. K. Padmanabhan, S. Singh et al., "Role of ABC transporter MRPA, $\gamma$-glutamylcysteine synthetase and ornithine decarboxylase in natural antimony-resistant isolates of Leishmaniadonovani," Journal of Antimicrobial Chemother$a p y$, vol. 59, no. 2, pp. 204-211, 2007.

[23] J. Walker, R. Gongora, J. J. Vasquez et al., "Discovery of factors linked to antimony resistance in Leishmaniapanamensis through differential proteome analysis," Molecular and Biochemical Parasitology, vol. 183, no. 2, pp. 166-176, 2012.

[24] R. Mukhopadhyay, S. Dey, N. Xu et al., "Trypanothione overproduction and resistance to antimonials and arsenicals in Leishmania," Proceedings of the National Academy of Sciences of the United States of America, vol. 93, no. 19, pp. 10383-10387, 1996.

[25] J. I. Manzano, R. García-Hernández, S. Castanys, and F. Gamarro, "A new ABC half-transporter in Leishmaniamajor is involved in resistance to antimony," Antimicrobial Agents and Chemotherapy, vol. 57, no. 8, pp. 3719-3730, 2013.

[26] H. Denton, J. C. McGregor, and G. H. Coombs, "Reduction of anti-leishmanial pentavalent antimonial drugs by a parasitespecific thiol-dependent reductase, TDR1," Biochemical Journal, vol. 381, no. 2, pp. 405-412, 2004.

[27] B. Gourbal, N. Sonuc, H. Bhattacharjee et al., "Drug uptake and modulation of drug resistance in Leishmania by an aquaglyceroporin," Journal of Biological Chemistry, vol. 279, no. 30, pp. 31010-31017, 2004.

[28] A. Mukherjee, S. Boisvert, R. L. do Monte-Neto et al., "Telomeric gene deletion and intrachromosomal amplification in antimony resistant Leishmania," Molecular Microbiology, vol. 88, no. 1, pp. 189-202, 2013.

[29] A. Kumar, B. Sisodia, P. Misra, S. Sundar, A. K. Shasany, and A. Dube, "Proteome mapping of overexpressed membraneenriched and cytosolic proteins in sodium antimony gluconate (SAG) resistant clinical isolate of Leishmaniadonovani," British Journal of Clinical Pharmacology, vol. 70, no. 4, pp. 609-617, 2010.

[30] K. El Fadili, J. Drummelsmith, G. Roy, A. Jardim, and M. Ouellette, "Down regulation of KMP-11 in Leishmaniainfantum axenic antimony resistant amastigotes as revealed by a proteomic screen," Experimental Parasitology, vol. 123, no. 1, pp. 5157, 2009.

[31] M. Khadem Erfan, M. Mohebali, E. Kazemi-Rad et al., "Down regulation of Calcineurin gene is associated with Glucantime resistance in Leishmaniainfantum," Iranian Journal of Parasitology, vol. 8, no. 3, pp. 359-366, 2013.

[32] R. t'Kindt, R. A. Scheltema, A. Jankevics et al., "Metabolomics to unveil and understand phenotypic diversity between pathogen populations," PLOS Neglected Tropical Diseases, vol. 4, no. 11, Article ID e904, 2010.

[33] R. Mukhopadhyay, S. Mukherjee, B. Mukherjee et al., "Characterisation of antimony-resistant Leishmaniadonovani isolates: biochemical and biophysical studies and interaction with host cells," International Journal for Parasitology, vol. 41, no. 13-14, pp. 1311-1321, 2011.

[34] M. Berg, M. Vanaerschot, A. Jankevics et al., "Metabolic adaptations of Leishmaniadonovani in relation to differentiation, drug resistance and drug pressure," Molecular Microbiology, vol. 90, no. 2, pp. 428-442, 2013.

[35] G. Canuto, E. A. Castilho-Martins, M. Tavares et al., "CEESI-MS metabolic fingerprinting of Leishmania resistance to antimony treatment," Electrophoresis, vol. 33, no. 12, pp. 19011910, 2012.

[36] M. Ouellette, J. Drummelsmith, and B. Papadopoulou, "Leishmaniasis: Drugs in the clinic, resistance and new developments," Drug Resistance Updates, vol. 7, no. 4-5, pp. 257-266, 2004.

[37] M. Pourshafie, S. Morand, A. Virion, M. Rakotomanga, C. Dupuy, and P. M. Loiseau, "Cloning of S-adenosylL-methionine:C-24- $\Delta$-sterol-methyltransferase (ERG6) from Leishmaniadonovani and characterization of mRNAs in wildtype and amphotericin B-resistant promastigotes," Antimicrobial Agents and Chemotherapy, vol. 48, no. 7, pp. 2409-2414, 2004.

[38] L. Lachaud, N. Bourgeois, M. Plourde, P. Leprohon, P. Bastien, and M. Ouellette, "Parasite susceptibility to amphotericin B in failures of treatment for visceral leishmaniasis in patients coinfected with HIV type 1 and Leishmaniainfantum," Clinical Infectious Diseases, vol. 48, no. 2, pp. e16-e22, 2009.

[39] C. di Giorgio, F. Faraut-Gambarelli, A. Imbert, P. Minodier, M. Gasquet, and H. Dumon, "Flow cytometric assessment of amphotericin B susceptibility in Leishmaniainfantum isolates from patients with visceral leishmaniasis," Journal of Antimicrobial Chemotherapy, vol. 44, no. 1, pp. 71-76, 1999.

[40] P. Srivastava, V. K. Prajapati, M. Rai, and S. Sundar, "Unusual case of resistance to amphotericin $B$ in visceral leishmaniasis in a region in India where leishmaniasis is not endemic," Journal of Clinical Microbiology, vol. 49, no. 8, pp. 3088-3091, 2011.

[41] B. Purkait, A. Kumar, N. Nandi et al., "Mechanism of amphotericin B resistance in clinical isolates of Leishmaniadonovani," Antimicrobial Agents and Chemotherapy, vol. 56, no. 2, pp. 10311041, 2011.

[42] A. K. Singh, B. Papadopoulou, and M. Ouellette, "Gene amplification in amphotericin B-resistant Leishmania tarentolae," Experimental Parasitology, vol. 99, no. 3, pp. 141-147, 2001.

[43] M. Basselin, F. Lawrence, and M. Robert-Gero, "Pentamidine uptake in Leishmaniadonovani and Leishmaniaamazonensis promastigotes and axenic amastigotes," Biochemical Journal, vol. 315, pp. 631-634, 1996.

[44] A. C. Coelho, N. Messier, M. Ouellette, and P. C. Cotrim, "Role of the ABC transporter PRP1 (ABCC7) in pentamidine resistance in Leishmania amastigotes," Antimicrobial Agents and Chemotherapy, vol. 51, no. 8, pp. 3030-3032, 2007.

[45] P. G. Bray, M. P. Barrett, S. A. Ward, and H. P. De Koning, "Pentamidine uptake and resistance in pathogenic protozoa: past, present and future," Trends in Parasitology, vol. 19, no. 5, pp. 232-239, 2003.

[46] B. Papadopoulou, C. Kündig, A. Singh, and M. Ouellette, "Drug resistance in Leishmania: similarities and differences to other organisms," Drug Resistance Updates, vol. 1, no. 4, pp. 266-278, 1998.

[47] A. C. Coelho, S. M. Beverley, and P. C. Cotrim, "Functional genetic identification of PRP1, an ABC transporter superfamily member conferring pentamidine resistance in Leishmania 
major," Molecular and Biochemical Parasitology, vol. 130, no. 2, pp. 83-90, 2003.

[48] M. Basselin, H. Denise, G. H. Coombs, and M. P. Barrett, "Resistance to pentamidine in Leishmania mexicana involves exclusion of the drug from the mitochondrion," Antimicrobial Agents and Chemotherapy, vol. 46, no. 12, pp. 3731-3738, 2002.

[49] M. Basselin, F. Lawrence, and M. Robert-Gero, "Altered transport properties of Pentamidine-resistant Leishmaniadonovani and Leishmaniaamazonensis promastigotes," Parasitology Research, vol. 83, no. 5, pp. 413-418, 1997.

[50] A. Mukherjee, P. K. Padmanabhan, M. H. Sahani, M. P. Barrett, and R. Madhubala, "Roles for mitochondria in pentamidine susceptibility and resistance in Leishmaniadonovani," Molecular and Biochemical Parasitology, vol. 145, no. 1, pp. 1-10, 2006.

[51] M. Basselin, M. Badet-Denisot, F. Lawrence, and M. RobertGero, "Effects of Pentamidine on polyamine level and biosynthesis in wild-type, Pentamidine-treated and Pentamidineresistant Leishmania," Experimental Parasitology, vol. 85, pp. 274-282, 1997.

[52] D. B. Liarte and S. M. F. Murta, "Selection and phenotype characterization of potassium antimony tartrate-resistant populations of four New World Leishmania species," Parasitology Research, vol. 107, no. 1, pp. 205-212, 2010.

[53] M. Vanaerschot, S. de Doncker, S. Rijal, L. Maes, J. C. Dujardin, and S. Decuypere, "Antimonial resistance in Leishmaniadonovani is associated with increased in vivo parasite burden," PLoS ONE, vol. 6, no. 8, Article ID e23120, 2011.

[54] K. Ait-Oudhia, E. Gazanion, B. Oury, B. Vergnes, and D. Sereno, "The fitness of antimony-resistant Leishmania parasites: Lessons from the field," Trends in Parasitology, vol. 27, no. 4, pp. 141-142, 2011.

[55] F. Real, R. O. Vidal, M. F. Carazzolle et al., "The genome sequence of Leishmania (Leishmania) amazonensis: functional annotation and extended analysis of gene models," DNA Research, vol. 20, no. 6, pp. 567-581, 2013.

[56] C. S. Peacock, K. Seeger, D. Harris et al., "Comparative genomic analysis of three Leishmania species that cause diverse human disease," Nature Genetics, vol. 39, no. 7, pp. 839-847, 2007.

[57] R. A. Scheltema, S. Decuypere, R. T'Kindt, J.-C. Dujardin, G. H. Coombs, and R. Breitling, "The potential of metabolomics for Leishmania research in the post-genomics era," Parasitology, vol. 137, no. 9, pp. 1291-1302, 2010.

[58] D. Paape and T. Aebischer, "Contribution of proteomics of Leishmania spp. to the understanding of differentiation, drug resistance mechanisms, vaccine and drug development," Journal of Proteomics, vol. 74, no. 9, pp. 1614-1624, 2011.

[59] S. Sundar and P. L. Olliaro, "Miltefosine in the treatment of leishmaniasis: clinical evidence for informed clinical risk management," Therapeutics and Clinical Risk Management, vol. 3, no. 5, pp. 733-740, 2007.

[60] A. Khatami, A. Firooz, F. Gorouhi, and Y. Dowlati, "Treatment of acute Old World cutaneous Leishmaniasis: a systemic review of the randomized controlled trials," Journal of the American Academy of Dermatology, vol. 57, no. 2, pp. 335.el-335.e29, 2007.

[61] L. O. Neves, A. C. Talhari, E. P. Gadelha et al., "A randomized clinical trial comparing meglumine antimoniate, pentamidine and amphotericin B for the treatment of cutaneous leishmaniasis by Leishmania guyanensis," Anais Brasileiros de Dermatologia, vol. 86, no. 6, pp. 1092-1101, 2011.

[62] M. Maharjan, S. Singh, M. Chatterjee, and R. Madhubala, "Role of aquaglyceroporin (AQP1) gene and drug uptake in antimonyresistant clinical isolates of Leishmaniadonovani," The American
Journal of Tropical Medicine and Hygiene, vol. 79, no. 1, pp. 6975, 2008. 

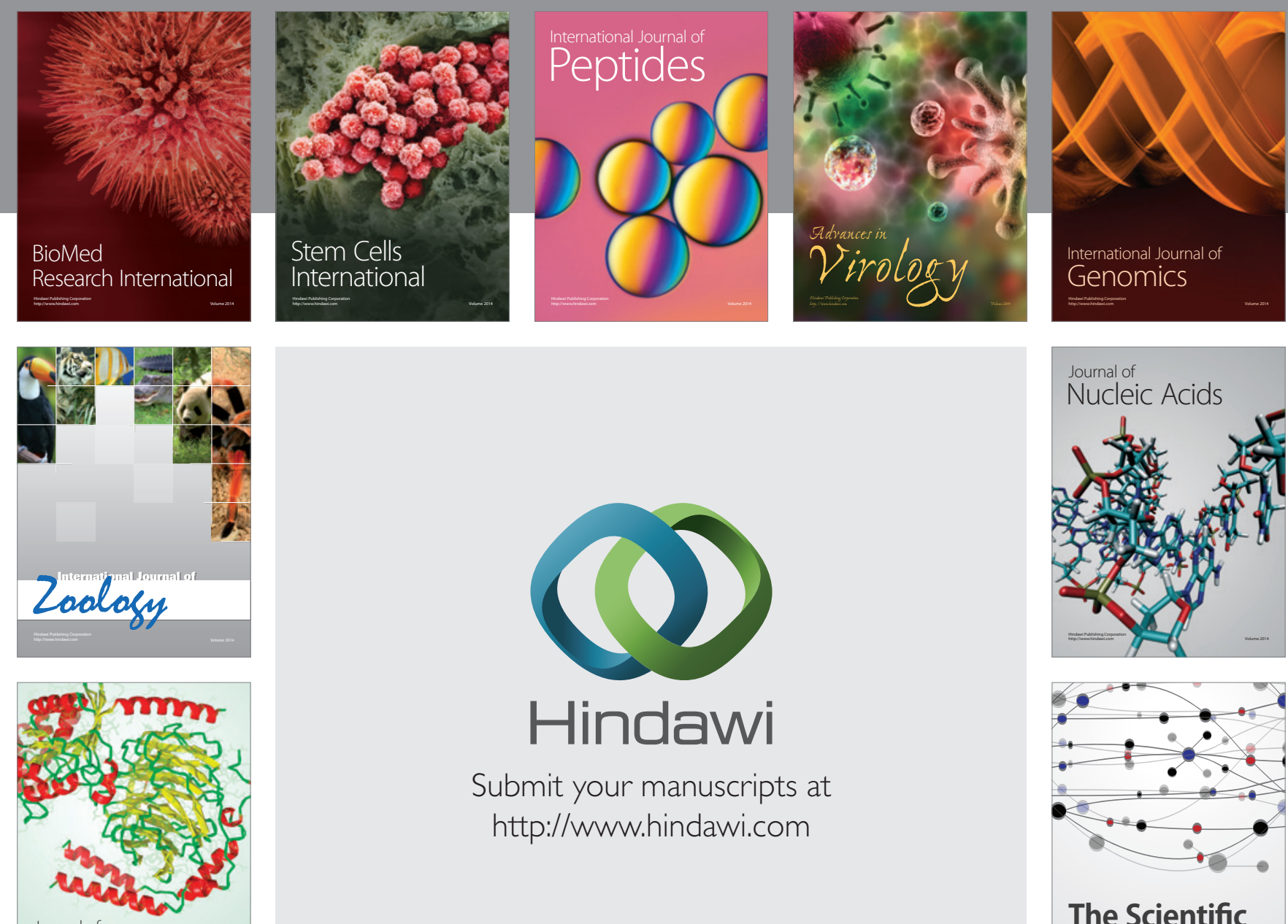

Submit your manuscripts at

http://www.hindawi.com

Journal of
Signal Transduction
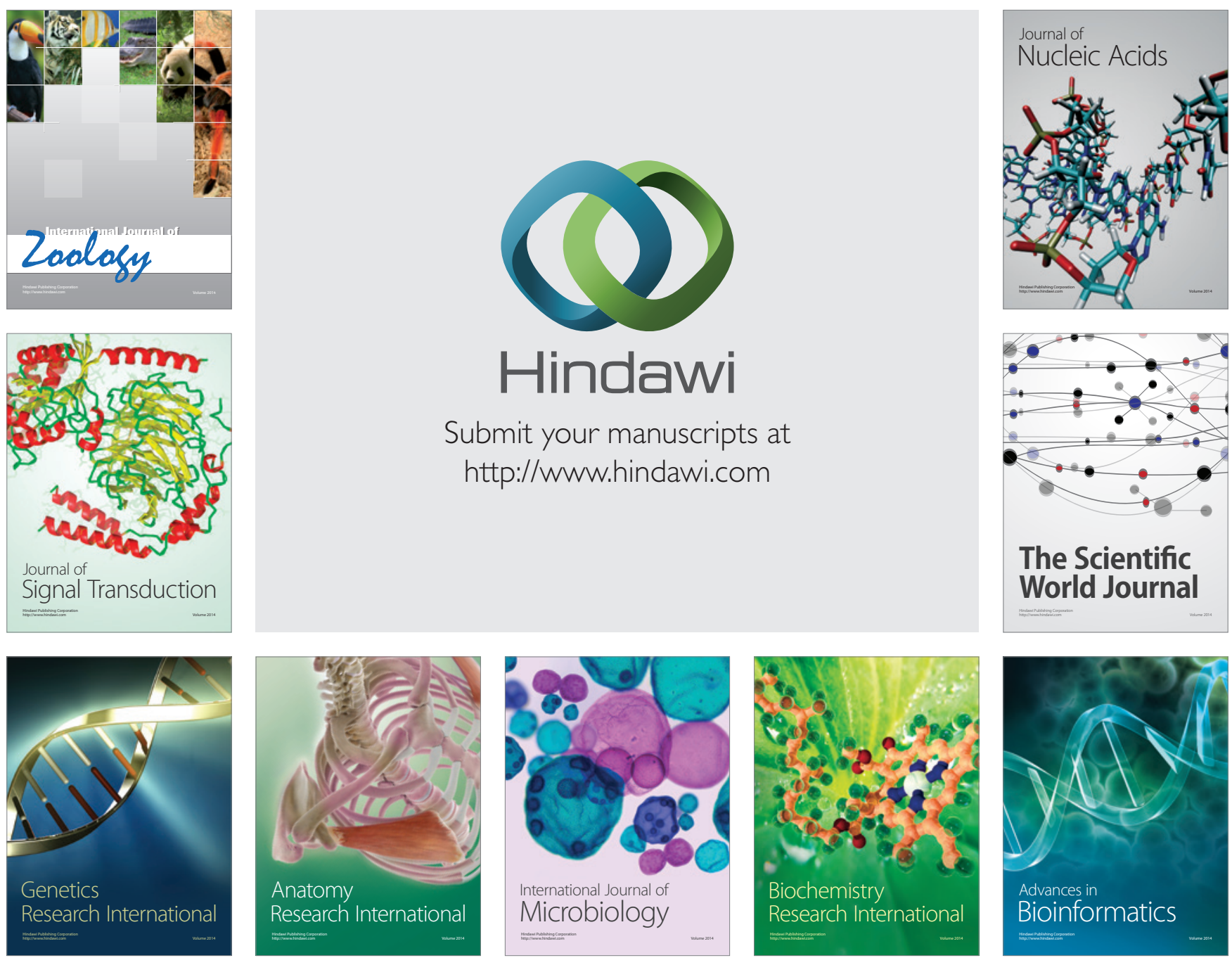

The Scientific World Journal
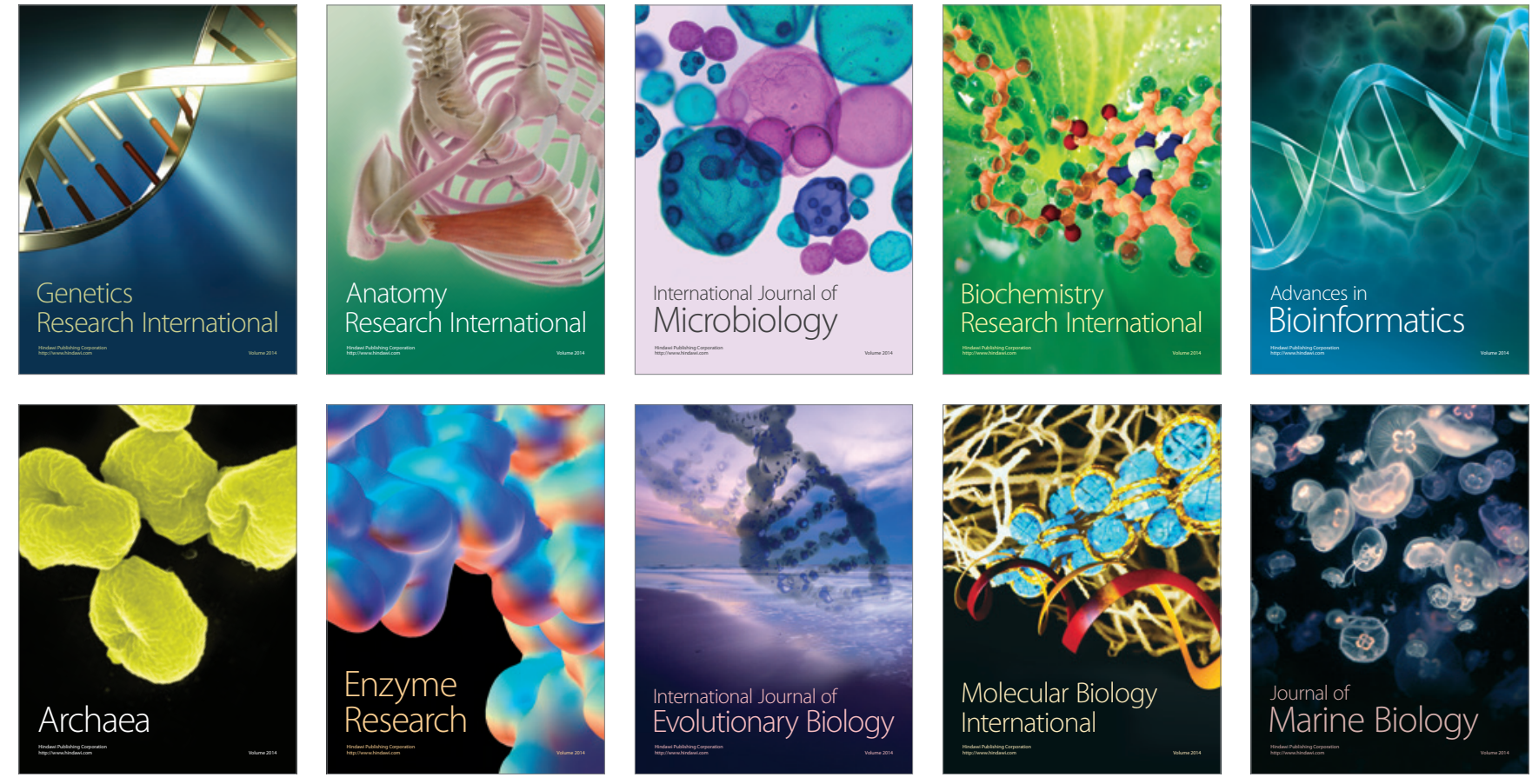